\title{
Berufssituation der Hebammen in Einrichtungen mit und ohne hebammengeleitete Geburtshilfe in einem Schweizer Kanton
}

Susanne Grylka-Baeschlin ${ }^{1}$, Barbara Borner ${ }^{1}$, Jessica Pehlke-Milde ${ }^{1}$

${ }^{1}$ Forschungsstelle Hebammenwissenschaft, Institut für Hebammen, Zürcher Hochschule für Angewandte Wissenschaften, Technikumstrasse 81, 8401 Winterthur, Schweiz

Korrespondierende Autorin: Susanne Grylka-Baeschlin, Forschungsstelle Hebammenwissenschaft, Institut für Hebammen, Zürcher Hochschule für Angewandte Wissenschaften, Technikumstrasse 81, 8401 Winterthur, Schweiz, E-Mail:

Susanne.Grylka@zhaw.ch, Tel. +4158934 4377 


\section{Deutsches Abstract}

Einleitung: Verglichen mit herkömmlichen Betreuungsmodellen bietet die hebammengeleitete Geburtshilfe den Hebammen mehr Möglichkeiten ihre Kompetenzen einzusetzen. Dies wirkt sich positiv auf ihre Berufszufriedenheit aus. Ziel dieser Erhebung war eine Übersicht über die Berufssituation der Hebammen in den Geburtenabteilungen eines Schweizer Kantons zu erlangen und Einrichtungen mit und ohne hebammengeleitete Geburtshilfe zu vergleichen.

Methodik: Ein Online-Fragebogen wurde literaturbasiert entwickelt. Alle 17 Institutionen des Kantons Zürich, die über eine Geburtenabteilung verfügten, waren teilnahmeberechtigt. Die Daten wurden mit Stata 15 deskriptiv ausgewertet.

Ergebnisse: 16 Geburtenabteilungen (94.1\%) nahmen teil: 12 öffentliche Kliniken, 2 Privatkliniken und 2 Geburtshäuser. Insgesamt 5 Einrichtungen (31.3\%) boten hebammengeleitete Geburten an oder waren Geburtshäuser. In Institutionen mit hebammengeleiteter Geburtshilfe kannten sich die Frauen und die Hebamme häufiger schon vor Aufnahme zur Geburt als in solchen ohne $(60.0 \%$ vs $9.1 \%, p=0.063)$, es wurden weniger routinemässige Massnahmen durchgeführt (z.B. venöser Zugang: $20.0 \%$ vs $81.8 \%$, $\mathrm{p}=0.036$ ), die Hebammen hatten mehr Entscheidungskompetenzen sowie Verantwortung (z.B. selbständige Austrittsuntersuchung: $60.0 \%$ vs $9.1 \%, p=0.063$ ) und nahmen häufiger Supervisionen in Anspruch (60.0\% vs 9.1\%, $p=0.013$ ).

Fazit: Das Fördern von hebammengeleiteten Betreuungsmodellen erhöht die Kontinuität in der Betreuung, senkt das Einsetzen von routinemässigen Massnahmen und fördert die eigenverantwortliche Übernahme von Aufgaben. 


\section{English Abstract}

\section{Occupational situation of midwives in institutions with and without midwife-led care in in a Swiss Canton}

Introduction: Compared to traditional models of care, midwife-led models of care provide better opportunities for midwives to make use of their full scope of practice. This has consequences for their professional satisfaction. The aim of this survey was to gain an overview of the occupational situation of midwives in the maternity units of a Swiss canton and to compare institutions with and without midwife-led care.

Methods: An online-survey was developed based on a literature search. All 17 institutions with maternity units in the canton of Zurich were eligible to participate. Data was analysed descriptively using Stata 15.

Results: 16 maternity units $(94.1 \%)$ participated in the survey: 12 public hospitals, 2 private hospitals and 2 midwifery-led birthplaces. A total of 5 units (31.3\%) provided midwife-led births or were birthplaces. In institutions providing midwife-led care, women and midwives were more likely to know each other before admission to birth $(60.0 \%$ vs $9.1 \%, p=0.063)$, less routine interventions were carried out (e.g. venous access: $20.0 \%$ vs $81.8 \%, p=0.036$ ), midwives had more decision-making competences as well as responsibility (e.g. autonomous discharge check-up: $60.0 \%$ vs $9.1 \%, p=0.063)$ and used supervisions more often $(60.0 \%$ vs $9.1 \%, p=0.013)$.

Conclusion: Fostering midwife-led models of care increases continuity of care and decreases the application of routine interventions. It also fosters the independent assumption of tasks. 


\section{Condensed Content}

Introduction: Midwives working in midwife-led models of care have more responsibility and are more satisfied than those working in traditional care models. Midwife-led models of care assist physiological processes and foster continuity of care as well as women-centred care. In Switzerland, physiological births can be midwife-led. However, there is a lack of knowledge about the occupational situation of midwives in clinical practice and the implementation of midwife-led care. The aim of this survey was therefore to gain an overview of midwives' occupational situation in a Swiss canton and to compare institutions with and without midwife-led care.

Methods: An online-survey about midwife-led continuity of care and the promotion of physiological birth was developed based on a literature search. All 17 institutions with maternity units in the canton of Zurich were eligible to participate. One person per institution with a management role was assigned by the maternity unit itself to complete the questionnaire. Data was analysed descriptively and study groups were compared with Fisher's exact tests using Stata 15.

Results: Out of 17 maternity units, 16 participated in the survey (response rate: $94.1 \%$ ): 12 public hospitals, 2 private hospitals and 2 midwifery-led birthplaces. A total of 5 units $(31.3 \%)$ offered officially or unofficially midwife-led births or were birthplaces. In institutions providing midwife-led care, women and midwives were more likely to know each other before admission for labour and birth $(60.0 \%$ vs $9.1 \%, p=0.063)$. Less routine intrapartum interventions were carried out in institutions with midwife-led care compared to those without (e.g. venous access: $20.0 \%$ vs $81.8 \%$, $p=0.036$; blood sampling for laboratory: $40.0 \%$ vs $90.9 \%, p=0.063$; routine oxytocin application for placenta: $0 \%$ vs $54.6 \%, p=0.058$ ) and midwives had more decision-making competencies as well as responsibility (e.g. autonomous discharge check-up: $60.0 \%$ vs $9.1 \%, p=0.063)$. The range of midwifery-specific further training was similar $(100 \%$ vs $72.7 \%, p=0.509)$ but the attendance at those trainings was significantly higher in units with midwife-led births than in those without (100\% vs $25.0 \%$, $p=0.021$ very well attended). Supervisions to reflect team dynamics and conflicts in moderated discussions were also more often used in units providing midwife-led care $(60.0 \%$ vs $9.1 \%, p=0.013)$.

Discussion: Despite being a political issue, midwife-led births are still in the minority in the canton of Zurich. Although the study sample was very small and three institutions recorded only a small proportion of midwife-led births, significant differences and those with a p-values between 0.05 and 0.10 between institution with and without midwife-led care were found regarding continuity of care, routine intrapartum interventions and midwives' competencies. Additionally, midwives working in units with midwife-led births show better attendance at further training courses and use more often supervisions. It seems that accepting more responsibilities leads to a need for greater knowledge and professional support. Limitations of this study were the small sample and the fact that only one person per institution completed the questionnaire. Individual midwives and other persons might have answered differently. However, the participation rate of over $90 \%$ is a strength of this study. Future research should investigate the occupational situation of midwives in larger areas.

Implementation in clinical practice: Midwives and parturients could profit from the promotion if midwife-led care, because it increases continuity of care and decreases the application of routine interventions. It also facilitates the use of midwives' full scope of practice. 


\section{Einleitung}

Hebammen, die in Einrichtungen mit hebammengeleiteter Geburtshilfe beschäftigt sind, arbeiten selbständiger und eigenverantwortlicher als ihre Kolleginnen in herkömmlichen Geburtenabteilungen [1,2]. Studien zeigen, dass Hebammen in hebammengeleiteten Betreuungsmodellen zufriedener sind, erweiterte Kompetenzen entwickeln und ein geringeres Risiko für eine Burnout-Erkrankung aufweisen [3-6]. Wenn Hebammen mit ihrer Arbeit zufriedener sind, verbleiben sie zudem länger im Beruf, was wiederum dem Fachkräftemangel entgegen wirkt [7].

Hebammengeleitete Betreuungsmodelle beinhalten das Fördern der physiologischen Prozesse und der Betreuungskontinuität in der peripartalen Phase [1,2]. Die Verantwortung liegt vollumfänglich bei der betreuenden Hebamme, welche die Bedürfnisse der Nutzerinnen in den Mittelpunkt stellt. Forschungsergebnisse stützen weltweit den Ansatz hebammengeleiteter Versorgungsmodelle, da diese im Vergleich zur herkömmlichen Betreuung mit vergleichbar guten geburtshilflichen Outcomes, weniger Interventionen, niedrigeren Kosten und einer erhöhten Zufriedenheit der Frauen einhergehen [2,8-11]. Die hebammengeleitete Geburtshilfe wird als zeitgemässes, effektives und sichereres Konzept betrachtet, das sowohl für die Gesundheit der Mütter und ihrer Kinder, als auch berufspolitisch, ökonomisch und gesellschaftlich relevant ist [1].

Die Berufssituation von Hebammen hängt stark von ihrem Arbeitsumfeld ab $[2,12]$. In einer integrativen Literaturübersicht zeigten Bloxsome et al. [7], dass gute Beziehungen zu den Arbeitskolleginnen, Unterstützung durch die leitenden Personen, die Möglichkeit, Beziehungen zu den betreuten Frauen aufzubauen und physiologische Prozesse zu fördern, sowie die Möglichkeit, ihr ganzes Berufsspektrum ausüben zu können, Aspekte sind, welche die Berufsverweildauer von Hebammen erhöhen. Beruflicher Stress hingegen ist mit einer niedrigeren Arbeitszufriedenheit und einer kürzeren Verweildauer assoziiert [13]. Eissler und Jerg-Bretzke [12] zeigten in einer Schweizer Querschnittstudie, dass sich belastende Faktoren je nach Arbeitsumfeld unterscheiden. So empfinden klinisch tätige Hebammen das hohe Arbeitspensum und Arbeitsunterbrechungen sowie fehlende Wertschätzung durch Ärzte und Ärztinnen als besonders belastend. In der Freiberuflichkeit leiden die Hebammen vor allem unter der Vereinbarkeit von Beruf und Familie und unter der Bereitschaftszeit [12].

Hebammen in der Schweiz sind qualifiziert, Frauen und Kinder während Schwangerschaft, Geburt, Wochenbett und Stillzeit eigenständig zu begleiten und im Falle von Abweichungen vom physiologischen Verlauf andere Fachpersonen hinzuzuziehen [14]. Die Schweizer Hebammenausbildung befindet sich seit 2008 auf Hochschulniveau [15]. Durch die Akademisierung der Ausbildung stieg das Niveau der erworbenen Kompetenzen und die 
Hebammen sind aufgefordert, wissenschaftliche Erkenntnisse zu reflektieren und ihr praktisches Handeln nach evidenzbasiertem Wissen auszurichten [16,17]. In hebammengeleiteten Betreuungsmodellen, in denen Hebammen eigenständig arbeiten und eine hohe Verantwortung tragen [2], können sie die erworbenen Kompetenzen einsetzen. In 2015 stellten Aubry et al. [1] jedoch fest, dass die meisten Hebammen in der Schweiz in herkömmlichen Betreuungsmodellen beschäftigt sind, da die hebammengeleitete Geburtshilfe bisher nur vereinzelt eingeführt und von werdenden Müttern wenig genutzt wurde $[1,18]$. Eine Westschweizer Studie hingegen zeigte, dass sowohl Gesundheitsfachpersonen, als auch schwangere Frauen hebammengeleiteten Versorgungsmodellen offen gegenüber stehen [18]. In den letzten Jahren entwickelten sich diese Betreuungsmodelle in der Schweiz zu einem politisch aktuellen Thema $[1,19]$. Sowohl im Kanton Bern als auch im Kanton Zürich gab es parlamentarische Vorstösse zur Stärkung der hebammengeleiteten Geburtshilfe $[1,19]$. Dabei wurde festgestellt, dass es in anderen europäischen Ländern wie den Niederlanden, Grossbritannien und Deutschland schon früher Bestrebungen gab, physiologische Geburten durch das Stärken von Hebammenversorgungsmodellen zu fördern. Bisher ist jedoch wenig bekannt, wie viele Schweizer Geburtenabteilungen hebammengeleitete Geburten anbieten und wie sich die Berufssituation der Hebammen, die in solchen Modellen arbeiten, von denjenigen, die in herkömmlichen Versorgungsmodellen tätig sind, unterscheidet.

Das Ziel dieser Erhebung war a) einen Überblick über die Berufssituation der Hebammen und die damit verbundenen Aspekten wie hebammengeleitete Geburtshilfe, Kontinuität in der Betreuung, Förderung der physiologischen Geburt und Entscheidungskompetenzen in den Geburtenabteilungen eines Schweizer Kantons zu erhalten; und b) Einrichtungen mit und ohne hebammengeleitete Geburtshilfe bezüglich dieser Aspekte zu vergleichen.

\section{Methodik}

\section{Studiendesign und Stichprobe}

Für die Bestandaufnahme der Berufssituation der Hebammen in einem Schweizer Kanton wurde eine Querschnittstudie in Form einer Online-Umfrage durchgeführt. Alle 17 Einrichtungen des Kantons Zürich in der Schweiz, die über eine Geburtenabteilung verfügten, waren teilnahmeberechtigt. Pro Institution wurde ein Fragebogen ausgefültt, entweder von der leitenden Hebamme oder der Pflegedienstleitung. Die Institutionen entschieden individuell, welche Person die Berufssituation der Hebammen am besten beurteilen konnte. Vor dem Versand der Umfrage wurde diese bei den leitenden Hebammen und den Pflegedienstleitungen der Frauenkliniken angekündigt und es wurden die Kontaktdaten der Person erfragt, die für das Ausfüllen des Fragebogens bestimmt wurde. 
Um die Teilnahmequote zu maximieren wurden mehrere Erinnerungen verschickt. Potentiell teilnehmende Institutionen erhielten schriftliche Studieninformationen und willigten durch das Ausfüllen der Online-Umfrage in die Studie ein. Die Studie wurde von Ethikkommission des Kantons Zürich als unbedenklich eingestuft (BASE-Nr. Req-2017-00133).

\section{Entwicklung des Fragebogens}

Für die Entwicklung des Fragebogens wurde eine Literaturanalyse zur hebammengeleiteten Geburtshilfe und Förderung der physiologischen Geburt durchgeführt. Basierend auf der Definitionen der hebammengeleiteten Geburtshilfe der Cochrane-Review von Sandall et al. [2] und Inhalten aus dem Expertinnenstandard zur Förderung der physiologischen Geburt [20] wurden Fragen erstellt. Zudem wurden Fragen aus der IST-Analyse des Handbuchs Hebammenkreisssaal [21] in die Umfrage integriert. Die Umfrage beinhaltete allgemeine Fragen (z.B. Art der Institution, Geburtenzahlen), Fragen zu Rahmenbedingungen und Ressourcen der Einrichtungen (z.B. Anzahl Hebammen, Räumlichkeiten, Versorgungsmodelle), zu Angeboten der Hebammen (z.B. Hebammensprechstunden, Kurse), zu den Bedürfnissen der Nutzerinnen, zur Kontinuität in der Betreuung, zur Förderung von physiologischen Geburten, zu Entscheidungskompetenzen der Hebammen, zu Fortbildungsmöglichkeiten für Hebammen und Fragen zu persönlichen Angaben der Person, die den Fragebogen ausfüllte. Für diese Umfrage wurden verschiedene Betreuungsmodelle wie folgt definiert: in der Pool-Betreuung wird die gebärende Frau von einer Hebamme aus einem ihr bekannten Team von zwei bis fünf Hebammen betreut. In der Caseload-Betreuung hingegen wird die gebärende Frau durch eine einzelne, ihr bekannte Hebamme versorgt. Zudem ist eine Eins-zu-eins-Betreuung gegeben, wenn die Hebamme nur eine Frau betreut.

Der Fragebogen wurde zuerst in Papierform von drei Hebammenexpertinnen beurteilt und entsprechend den Rückmeldungen angepasst. Anschliessend wurde der Online-Fragebogen von einer leitenden Hebamme im Ruhestand, einer stellvertretenden leitenden Hebamme in einem anderen Schweizer Kanton und einer Person, die nicht im Gesundheitswesen tätig war, pilotiert und weiter inhaltlich und sprachlich angepasst. Damit konnte sichergestellt werden, dass die Fragen für die Zielgruppe adäquat und verständlich waren, und sprachliche Ungereimtheiten korrigiert wurden [22].

\section{Datenaufbereitung und Analysen}

Die teilnehmenden Institutionen wurden in die Kategorien «mit hebammengeleiteten Geburten» und "ohne hebammengeleitete Geburten» unterteilt. Eine Einrichtung, die hebammengeleitete Geburten auf Anfrage anbot, wurde zur Gruppe «mit hebammengeleiteten Geburten» gezählt. Die Daten wurden entsprechend der Art und Verteilung der 
Variablen deskriptiv ausgewertet. Institutionen mit und ohne hebammengeleitete Geburtshilfe wurden aufgrund der kleinen Fallzahlen mit dem exakten Test nach Fisher verglichen. Ergebnisse mit $p<0.05$ wurden als signifikant betrachtet und solche mit $p<0.10$ aufgrund der kleinen Fallzahl in die Diskussion einbezogen. Die Analysen wurden mit Stata 15 durchgeführt (StataCorp, Tx, USA).

\section{Ergebnisse}

Von 17 teilnahmeberechtigten Institutionen nahmen 16 an der Umfrage teil (Rücklaufquote 94.1\%). Insgesamt füllten zwölf Leitungspersonen von Geburtenabteilungen (75.0\%), eine Co-Leitung der Frauenklinik (6.3\%), eine Pflegedienstleitung (6.3\%) sowie je ein Mitglied der Geschäftsleitung und der Ressortleitung Qualität und Geburtshilfe (je 6.3\%) den Fragebogen aus. Als höchsten Bildungsabschluss verfügten vier Personen (25.0\%) über ein Hebammendiplom oder höheren Fachschulabschluss, zehn (62.5\%) über einen Bachelorabschluss und zwei (12.5\%) über einen Masterabschluss.

Die teilnehmenden Institutionen

Unter den 16 teilnehmenden Institutionen befanden sich zwölf öffentliche Spitäler $(75.0 \%)$, zwei Privatkliniken (12.5\%) und zwei Geburtshäuser (12.5\%). Die Geburtenzahlen in 2016 lagen zwischen 203 und 2.962, wobei elf Institutionen (68.8\%) weniger als 1.000 Geburten, zwei Institutionen (12.5\%) zwischen 1.001 und 2.000 Geburten und drei Institutionen (18.8\%) mehr als 2.000 Geburten aufwiesen. Die prozentualen Anteile an Spontangeburten lagen zwischen $38.4 \%$ und $100 \%$, diejenigen von Instrumentalgeburten zwischen $0 \%$ und $16.1 \%$ und diejenigen von Sectiones caesarea zwischen 0\% und 56.9\%. Durchschnittlich verfügten die Einrichtung über 4.1 Gebärzimmer (Range 2-8). Davon hatten 55.9\% eine Badewanne (Range 1-4 Badewannen pro Institution).

\section{Personalsituation}

In den teilnehmenden Einrichtungen waren zwischen 9 und 46 Hebammen beschäftigt. Zudem arbeiteten in fünf Institutionen zwischen einer und drei Beleghebammen. In 15 Einrichtungen (93.8\%) wurden Hebammenstudierende ausgebildet. Es gab durchschnittlich 3.7 Hebammen pro Institution (Range 0-12), die weniger als zwei Jahre Berufserfahrung hatten. Dies entsprach durchschnittlich 15.5\% der Hebammen eines Teams (Range 044.4\%). In fünf Einrichtungen (31.3\%) arbeiteten die Hebammen regelmässig und in sieben weiteren (43.8\%) ausnahmsweise auf anderen Stationen. In allen fünf Institutionen, in denen Hebammen regelmässig auf anderen Abteilungen tätig waren, war dies in einem Rotationssystem organisiert. Die Hebammen arbeiteten zusätzlich zur Geburtenabteilung auf der Wochenbettstation, der Pränatalstation, der Gynäkologie und der Neonatologie. In elf 
Einrichtungen (68.8\%) wurde Personal eingesetzt, das die Hebammen entlastete. In fünf dieser Institutionen (45.5\%) waren dies Pflegehelferinnen, in drei (27.3\%) Fachangestellte Gesundheit, in vier (36.4\%) Sekretäre oder Sekretärinnen, in fünf (45.5\%) Raumpflegerinnen und in je einer Institution (9.1\%) Kinderpflegerinnen oder Stationshilfen.

\section{Hebammenbetreuung und -angebote}

In vier Einrichtungen (25.0\%) wurden offiziell hebammengeleitete Geburten angeboten, entweder in einem Hebammenkreisssaal oder als Geburtshaus. Die Einrichtungen verzeichneten 2016 zwischen 46 und 302 hebammengeleite Geburten. Eine Institution (6.3\%) bot hebammengeleitete Geburten nur auf Anfrage an und konnte die Anzahl der hebammengeleiteten Geburten nicht beziffern. In einer weiteren Institution (6.3\%) war ein Hebammenkreisssaal in Planung. Im Jahr 2016 wurden von insgesamt 17.368 Geburten in den teilnehmenden Institutionen 629 (3.6\%) als hebammengeleitet eingestuft. Der Anteil hebammengeleiteter Geburten lag in den beiden Geburtshäusern bei 100\% und in den beiden Einrichtungen, die sowohl hebammengeleitete als auch nicht hebammengeleitete Geburten anboten und dies beziffern konnten, bei 3.7\% bzw. 13.9\% aller Geburten. In Institutionen, die beides anboten, gab es weder eine räumliche noch eine personelle Trennung zwischen den üblichen und den hebammengeleiteten Kreisssälen. In zwei Einrichtungen mit hebammengeleiteten Geburten betreuten die Hebammen zudem im Jahr 2016 drei bzw. sieben Hausgeburten.

Die Hebammen boten in drei Institutionen (18.8\%) eine Poolbetreuung an, in der die gebärende Frau von einer bekannten Hebamme aus einem Team mit zwei bis fünf Hebammen betreut wurde (Institution mit hebammengeleiteten Geburten: 20.0\% vs Institution ohne hebammengeleitete Geburten: 18.2\%, $p=0.705$ ). Die Beleghebammen hingegen arbeiteten alle im Caseload-Betreuungsmodell, in dem die gebärende Frau ausschliesslich von einer ihr bekannten Hebamme betreut wird.

In 15 Institutionen (93.8\%) gab es eine Hebammensprechstunde (mit hebammengeleiteten Geburten: $100 \%$ vs ohne hebammengeleitete Geburten: $90.9 \%, p=0.687$ ). In einer Einrichtung ohne hebammengeleitete Geburtshilfe (6.8\%) war eine Hebammensprechstunde in Planung. In acht Einrichtungen (57.1\%; mit hebammengeleiteten Geburten: $60.0 \%$ vs ohne hebammengeleitete Geburten: $55.6 \%, p=0.657$ ) lag der Lead der Schwangerenvorsorge von Frauen, welche Hebammensprechstunden in Anspruch nahmen, bei der Hebamme, in zwei Institutionen (14.3\%; mit hebammengeleiteten Geburten: 20.0\% vs ohne hebammengeleitete Geburten: $11.1 \%, p=0.604)$ beim Arzt oder der Ärztin und in vier Einrichtungen (28.6\%; mit hebammengeleiteten Geburten: $20 . \%$ vs ohne 
hebammengeleitete Geburten: 33.3\%, $\mathrm{p}=0.545$ ) gab es keinen klaren Lead bzw. die Betreuung wurde alternierend von der Hebamme und vom Arzt oder der Ärztin durchgeführt.

Ein Geburtsplanungsgespräch, das schwangeren Frauen die Gelegenheit gibt, die bevorstehende Geburt zu besprechen und Fragen zu stellen, wurde in elf Institutionen (68.8\%; mit hebammengeleiteten Geburten: $100 \%$ vs ohne hebammengeleitete Geburten: $54.6 \%, p=0.433)$ angeboten. In 14 Institutionen (87.5\%; mit hebammengeleiteten Geburten: $80.0 \%$ vs ohne hebammengeleitete Geburten: $90.9 \%, p=0.542$ ) gab es eine Akupunktursprechstunde und sechs Einrichtungen (37.5\%; mit hebammengeleiteten Geburten: $60.0 \%$ vs ohne hebammengeleitete Geburten: $27.3 \%, p=0.267$ ) boten weitere durch Hebammen erbrachte Leistungen wie Schwangerenvorsorge ab Termin, Nachgespräche nach der Geburt, Hebammenberatung, Craniosakraltherapie und weitere an. Geburtsvorbereitungskurse durch Hebammen wurden in allen 16 (100\%) und Rückbildungsgymnastik durch Hebammen in zehn Einrichtungen (62.5\%; mit hebammengeleiteten Geburten: $80.0 \%$ vs ohne hebammengeleitete Geburten: $54.6 \%, p=0.084$ ) angeboten. Zudem gab es in neun Institutionen (56.3\%; mit hebammengeleiteten Geburten: $40.0 \%$ vs ohne hebammengeleitete Geburten: $45.5 \%, p=0.635$ ) weitere Kurse wie zum Beispiel Geburtsvorbereitungskurse im Wasser, Yoga, Säuglingspflegekurse, Geschwisterkurse, Babymassage oder Haptonomie.

In sechs Einrichtungen (37.5\%; mit hebammengeleiteten Geburten: $60.0 \%$ vs ohne hebammengeleitete Geburten: $27.3 \%, p=0.242$ ) wurden Eins-zu-eins-Betreuungen angeboten. Davon gaben zwei Institutionen (33.3\%; mit hebammengeleiteten Geburten: $66.7 \%$ vs ohne hebammengeleitete Geburten: $0 \%, p=0.200$ ) an, dass die Eins-zu-einsBetreuung während einer Schicht immer gewährleistet war, während in drei weiteren Einrichtungen (50.0\%; mit hebammengeleiteten Geburten: $33.3 \%$ vs ohne hebammengeleitete Geburten: 66.7\%, p=0.500) dies oft gewährleistet war.

\section{Bedürfnisse der schwangeren und gebärenden Frauen im Leitbild verankert}

In zwölf Institutionen (75.0\%; mit hebammengeleiteten Geburten: $60.0 \%$ vs ohne hebammengeleitete Geburten: 81.8\%, $\mathrm{p}=0.365$ ) gab es ein Leitbild oder eine Vision, in dem die Bedürfnisse der Nutzerinnen verankert waren. In neun der zwölf Einrichtungen (75.0\%; mit hebammengeleiteten Geburten: $100 \%$ vs ohne hebammengeleitete Geburten: $66.7 \%$, $p=0.382$ ), die ein Leitbild oder eine schriftlich verankerte Vision hatten, waren diese den Mitarbeitenden bekannt, in drei weiteren (25.0\%; mit hebammengeleiteten Geburten: 0\% vs ohne hebammengeleitete Geburten: $33.3 \%, p=0.382$ ) war dies nur teilweise der Fall. Spezielle Angebote für Migrantinnen gab es in drei Einrichtungen, alle ohne hebammengeleitete Geburtshilfe ( $27.3 \%$ vs $0 \%, p=0.295)$. In allen drei Institutionen war dies 
ein Dolmetscherdienst, in zwei Informationsmaterial in verschiedenen Sprachen und in einer spezielle Kurse für Migrantinnen.

\section{Kontinuität in der Betreuung}

In vier Einrichtungen (25.0\%) ging man davon aus, dass gebärende Frauen ihre betreuenden Hebammen grösstenteils schon vor dem Eintritt für die Geburt kannten (mit hebammengeleiteten Geburten: $60.0 \%$ vs ohne hebammengeleitete Geburten: $9.1 \%$, $p=0.063)$. Ebenfalls in vier Klinken $(25.0 \%)$ wurden die Wöchnerinnen grösstenteils von einer innen bekannten Hebamme oder einem innen bekanntem Hebammenteam betreut, ebenfalls häufiger in Institutionen mit hebammengeleiteter Geburtshilfe im Vergleich zu solchen ohne (60.0\% vs $9.1 \%, p=0.063)$. Weiter schätzten die Teilnehmenden in zwei Einrichtungen, dass die Wöchnerinnen zu Hause grösstenteils von einer innen bekannten Hebamme oder einem innen bekannten Hebammenteam betreut wurden (12.5\%; mit hebammengeleiteten Geburten: $20.0 \%$ vs ohne hebammengeleitete Geburten: $9.1 \%, p=0.542)$. Es gab keinen Unterschied im Anteil an Institutionen, die Angebote oder Strukturen (z.B. spezielle Tools in der elektronischen Akte, interdisziplinäre Rapporte) besitzen, welche die Informationsweitergabe zwischen Schwangerenvorsorge, Geburt und Wochenbettbetreuung innerhalb der Institution förderten (mit hebammengeleiteten Geburten: $66.7 \%$ vs ohne hebammengeleitete Geburten: 72.7\%, p=0.670, Antwort mit 12.5\% fehlenden Werten). Prozesse oder Angebote, welche die Kontinuität in der Betreuung an den Schnittstellen zwischen der ambulanten Schwangeren- bzw. Wochenbettbetreuung ausserhalb und der Betreuung innerhalb ihrer Institution fördern (z.B. Austauschtreffen zwischen Klinik und freipraktizierenden Hebammen sowie Mütter- und Väterberatungen) waren in Einrichtungen mit hebammengeleiteter Geburtshilfe häufiger als in solchen ohne $(80.0 \%$ vs $36.4 \%$, $p=0.141)$, der Unterschied war jedoch nicht signifikant.

\section{Physiologische Geburt}

Massnahmen zur Förderung der physiologischen Geburt wie der Einbezug der Frauen bei der Schichtübergabe, die Möglichkeiten, die Geburtsposition frei zu wählen oder im Wasser zu gebären sowie das Fördern einer grösstmöglichen Mobilität der Frauen während des Gebärens unterschieden sich nicht signifikant zwischen Institutionen mit oder ohne hebammengeleiteter Geburtshilfe (Tabelle 1). Routinemässige Massnahmen bei physiologischen Geburten wurden in Institutionen mit hebammengeleiteter Geburtshilfe weniger häufig angewendet als in solchen ohne. Die p-Werte dieser Unterschiede lagen jedoch grösstenteils zwischen 0.05 und 0.10 (Legen eines intravenösen Zugangs: mit hebammengeleiteten Geburten: $20.0 \%$ vs ohne hebammengeleitete Geburten: $81.8 \%$, $p=0.036$; Blutentnahme für Laboruntersuchung: $40.0 \%$ vs $90.9 \%, p=0.063$; 
Kardiotokographie (CTG) bei Aufnahme: $60.0 \%$ vs 100\%, p=0.083; Aufnahmeuntersuchung Arzt/Ärztin: $0 \%$ vs $54.6 \%, p=0.058$; synthetisches Oxytocin in der Plazentarperiode $0 \%$ vs $54.6 \%, p=0.058$, Tabelle 1).

\section{Entscheidungskompetenzen im Kreisssaal}

In zwölf Einrichtungen (75.0\%) gab es klare Absprachen zwischen Hebammen und Ärzten sowie Ärztinnen bezüglich der Verantwortlichkeiten im Kreisssaal (mit hebammengeleiteten Geburten: $80.0 \%$ vs ohne hebammengeleitete Geburten: $72.7 \%, p=0.635)$. In fünf dieser elf Institutionen (45.5\%) wurden diese Absprachen schriftlich festgehalten (mit hebammengeleiteten Geburten: $33.3 \%$ vs ohne hebammengeleitete Geburten: 50.0\%, $p=0.576$ ). Die Kompetenz, selbständig und eigenverantwortlich Entscheidungen zu treffen, wurde in dieser Erhebung sehr detailliert abgefragt (Tabelle 2). Signifikante Unterschiede und solche mit pWerten zwischen 0.05 und 0.10 gab es bei der Entscheidung, ob Gebärende in der Latenzphase $(p=0.013)$ und Gebärende mit vorzeitigem Blasensprung nach Hause zurückkehren können $(p=0.004)$, bei der Durchführung einer Ultraschalluntersuchung $(p=0.072)$, der Mobilität unter der Geburt $(p=0.093)$, einem Entspannungsbad $(p=0.063)$ und dem Beginn einer Wehenstimulation mit Oxytocin $(p=0.098)$. Der hauptsächliche Unterschied war, dass in Einrichtungen mit hebammengeleiteter Geburtshilfe häufiger die Hebamme und in solchen ohne hebammengeleitete Geburten häufiger der Arzt oder die Ärztin in die Entscheidungsfindung miteinbezogen wurde (mit oder ohne Einbezug der Gebärenden). Bei Entscheidungen, ob die Gebärende in der Latenzphase zu Hause bleiben konnte oder auf der Geburtenabteilung aufgenommen wurde, gab es keinen signifikanten Unterschied zwischen Einrichtungen mit und ohne hebammengeleitete Geburtshilfe. Auch beim Anwenden einer intermittierenden oder kontinuierliche Kardiotokografie, beim Verabreichen von Spasmolytika, Opioide, Periduralanästhesie oder Lachgas oder beim Steigern einer Wehenstimulation mit Oxytocin gab es keinen Unterschied.

\section{Selbständige und eigenverantwortliche Tätigkeiten der Hebammen}

Aufgaben auf der Geburtenabteilung, wie das Erheben der Anamnese der Frau, das klinische Assessment für die Aufnahme zur Geburt, das Durchführen einer Amniotomie oder eines Dammschutzes, das Schneiden einer Episiotomie, das Nähen von Dammverletzungen und das Durchführen des Neugeborenenstatus wurden in Einrichtungen mit hebammengeleiteter Geburtshilfe häufiger «immer»/«meistens» selbständig und eigenverantwortlich durch Hebammen durchgeführt, die Unterschiede waren jedoch nicht signifikant (Tabelle 3). Das Anwenden von alternativen Massnahmen sowie das Erheben des Apgar-Scores lag in allen Institutionen «immer»/«meistens» in den Händen der Hebammen. Die Verantwortung für die Austrittuntersuchung lag in Einrichtungen mit hebammengeleiteter Geburtshilfe 
häufiger bei den Hebammen als in solchen ohne (60.0\% vs $9.1 \%, p=0.063)$ (Tabelle 4). Die Hebammen waren in allen Einrichtungen verantwortlich für das Vermitteln einer ambulanten Wochenbettbetreuung.

\section{Supervisionen und Weiterbildungen}

Hebammen in Einrichtungen mit hebammengeleiteter Geburtshilfe nahmen signifikant häufiger Supervisionen in Anspruch (60.0\% vs 9.1\%, p=0.013). Obwohl nicht signifikant häufiger spezielle Weiterbildungsangebote für Hebammen angeboten wurden (100\% vs $72.7 \%, p=0.509$ ), wurden diese häufiger «sehr gut» besucht (Institutionen mit hebammengeleiteten Geburten: 100\% «sehr gut» besucht vs Institutionen ohne hebammengeleitete Geburten: $25.0 \%$ «sehr gut», $62.5 \%$ «grösstenteils gut» und $12.5 \%$ «teilweise gut» besucht, $p=0.021$ ).

\section{Diskussion}

In der vorliegenden Bestandsaufnahme wurde erstmals die Berufssituation von Schweizer Hebammen in Einrichtungen mit und ohne hebammengeleitete Geburtshilfe untersucht. Die verantwortlichen Personen in Einrichtungen mit hebammengeleiteter Geburtshilfe, welche den Fragebogen ausfüllten, schätzen die Rate an kontinuierlichen Betreuungssituationen höher und das Anwenden von routinemässigen Massnahmen bei physiologischen Geburten niedriger ein als solche in Einrichtungen ohne hebammengeleitete Geburtshilfe. Die Entscheidungsprozesse waren stärker durch die Hebammen und weniger durch Ärzte und Ärztinnen geprägt und die Hebammen waren häufiger verantwortlich für die Austrittuntersuchungen. Zudem nahmen sie häufiger Supervisionen in Anspruch, bei denen in moderierten Gesprächen die Teamdynamik und Konflikte reflektiert und diskutiert wurden. Auch die Teilnahme an hebammenspezifischen Weiterbildungsangeboten war in Institutionen mit hebammengeleiteter Geburtshilfe besser.

Obwohl hebammengeleitete Geburten im Kanton Zürich ein politisch aktuelles Thema sind $[1,19]$, hatten nur 3.6\% der Frauen, die in den teilnehmenden Einrichtungen geboren hatten, eine solche. Vermutlich ist der effektive Anteil aller hebammengeleiteten Geburten im Kanton Zürich jedoch grösser als derjenige der vorliegenden Bestandsaufnahme, da eine Institution die Anzahl hebammengeleiteter Geburten nicht beziffern konnte und Hausgeburten nicht Bestandteil dieser Erhebung waren. In der Statistik der frei praktizierenden Hebammen des Schweizerischen Hebammenverbandes wurde für 2016 ein gesamtschweizerischer Anteil von $4.8 \%$ hebammengeleiteten Geburten berechnet [23]. Darin sind jedoch Geburten in Einrichtungen, in denen angestellte Hebammen selbständig Geburten leiten, nicht enthalten und ein Vergleich der Ergebnisse der vorliegenden Studie mit gesamtschweizerischen Daten 
ist demnach nicht möglich. Es kann jedoch vermutet werden, dass hebammengeleitete Geburten im Kanton Zürich nach wie vor die Ausnahme darstellen.

Eine bessere Kontinuität in der Betreuung, weniger routinemässige Massnahmen bei physiologischen Geburten und mehr Entscheidungskompetenzen sind Ergebnisse, die im Rahmen der hebammengeleiteten Geburtshilfe zu erwarten waren und deren Definition entsprechen [1,2]. Erstaunlich ist jedoch, dass trotz der kleinen Stichprobe und trotz der Tatsache, dass ausser in den beiden Geburtshäusern nur ein kleiner Anteil der Geburten hebammengeleitet waren, einige Ergebnisse signifikant waren oder p-Werte zwischen 0.05 und 0.10 verzeichneten. Es kann deshalb vermutet werden, dass die hebammengeleitete Geburtshilfe in Einrichtungen Auswirkungen auf die Betreuungs- und demnach auch Berufssituation der angestellten Hebammen bezüglich den Aspekten Kontinuität, Anwendung von routinemässigen Massnahmen und Entscheidungskompetenzen mit sich bringen. Mehr Kontinuität in der Betreuung und damit bessere Möglichkeiten, eine Beziehung zu den Frauen aufzubauen sowie mehr Entscheidungskompetenzen, welche die Autonomie in der Berufsausübung erhöhen, stehen gemäss Bloxsome et al. [7] in einem positiven Zusammenhang mit der Berufsverweildauer der Hebammen. Es braucht jedoch grösser angelegte Studien, in denen die betreuenden Hebammen direkt befragt werden, um die genauen Zusammenhänge zwischen hebammengeleiteter Geburtshilfe und Berufsverweildauer aufzuzeigen.

Die häufigere Inanspruchnahme von Supervisionen und die zahlreichere Teilnahme an spezifischen Weiterbildungen in Einrichtungen mit hebammengeleiteter Geburtshilfe könnte darauf hindeuten, dass Hebammen, die in diesen Modellen arbeiten, ein grösseres Bedürfnis nach zusätzlichem Wissen und Unterstützung haben. Dies erstaunt nicht, da sie mehr Verantwortung tragen und ihre beruflichen Kompetenzen besser einsetzen können. Auch im Handbuch Hebammenkreisssaal [21] wird die Notwendigkeit für spezifische Weiterbildungen für Hebammen anerkannt und es wird empfohlen, für die Einführung der hebammengeleiteten Geburtshilfe einen Fortbildungsplan zu erstellen.

Die kleine Stichprobe war eine Limitation dieser Studie, welche eine Verallgemeinerung der Ergebnisse für andere Settings verunmöglicht. Unterschiede zwischen Institutionen mit und ohne hebammen-geleitete Geburtshilfe wären möglicherweise in einer grösseren Stichprobe eher signifikant.. Weitere Limitation sind, dass der Online-Fragebogen in jeder Einrichtung nur von einer leitenden Person ausgefüllt wurde und dass Sectio- und Einleitungsraten der Institutionen nicht verglichen werden konnten. Zu den Einleitungen wurden keine Daten erhoben und der Vergleich der Sectioraten war nicht sinnvoll, da die Geburtshäuser die Gebärenden zur Schnittentbindung in die Klinik verlegten und die angegebenen 0\% Anteile an Sectiones nicht aussagekräftig waren. Weiter war es im Rahmen dieser Publikation nicht 
möglich, die Evidenzen aller von Hebammen geleisteten Angebote aufzuarbeiten. Es ist zudem nicht auszuschliessen, dass Antworten anders ausgefallen wären, hätten andere Personen an der Umfrage teilgenommen. Die hohe Teilnahmequote von $94.1 \%$ hingegen ist eine Stärke der vorliegenden Bestandsaufnahme. Auch wenn die Ergebnisse nicht für die ganze Schweiz gültig sind, so sind sie doch regional für den Kanton Zürich aussagekräftig.

\section{Fazit für die Praxis}

Mit dem Fördern der hebammengeleiteten Geburtshilfe könnte erreicht werden, dass Hebammen in ihrem Berufsalltag mehr Kontinuität in der Betreuung erleben, weniger routinemässige Massnahmen anwenden und mehr Verantwortung und Entscheidungskompetenzen entsprechend ihrer Qualifikation wahrnehmen können. Erkenntnisse aus der wissenschaftlichen Literatur zeigen, dass dies die Arbeits- und Berufszufriedenheit von Hebammen steigern und möglicherweise die Berufsverweildauer verlängern könnte [2,7]. Die zahlreichere Teilnahme an spezifischen Weiterbildungen von Hebammen, die in hebammengeleiteten Modellen arbeiten, deutet darauf hin, dass in der beruflichen Praxis ein Weiterlernen notwendig ist. Es sollte geprüft werden, wie das Weiterbildungsangebot auch in Institutionen mit herkömmlichen Betreuungsmodellen attraktiver gestaltet werden könnte. Weitere Forschung in grösseren Stichproben und unter Einbezug der betreuenden Hebammen ist notwendig, um zu zeigen, ob und wie die kontinuierlichen und hebammengeleiteten Versorgungsmodelle die Berufszufriedenheit und die Berufsverweildauer nachhaltig verbessern können. 


\section{Referenzen}

1 Aubry, E., Cignacco, A. Hebammengeleitete Geburtshilfe im Kanton Bern Antwort auf das Postulat der Grossrätin Frau Natalie Imboden (126.2013), - Hebammengeleitete Geburtshilfe im Kanton Bern Antwort auf das Postulat der Grossrätin Frau Natalie Imboden (126.2013). Ein Expertinnen- und Expertenbericht. 2015; Im Internet: https://www.gesundheit.bfh.ch/fileadmin/_migrated/content_uploads/Grundlagenbericht_B erner_Fachhochschule_Fachbereich_Gesundheit.pdf; Stand: 20.02.2019

2 Sandall J, Soltani H, Gates S, Shennan A, Devane D. Midwife-led continuity models versus other models of care for childbearing women. Cochrane Database Syst Rev 2016; 4: CD004667. doi: 10.1002/14651858.CD004667.pub5

3 Collins CT, Fereday J, Pincombe J, Oster C, Turnbull D. An evaluation of the satisfaction of midwives' working in midwifery group practice. Midwifery 2010; 26: 435-441. doi: 10.1016/j.midw.2008.09.004

4 Cummins AM, Denney-Wilson E, Homer CSE. The experiences of new graduate midwives working in midwifery continuity of care models in Australia. Midwifery 2015; 31: 438-444. doi: 10.1016/j.midw.2014.12.01

5 Jepsen I, Mark E, Nøhr EA, Foureur M, Sørensen EE. A qualitative study of how caseload midwifery is constituted and experienced by Danish midwives. Midwifery 2016; 36: 61-69. doi: 10.1016/j.midw.2016.03.002

6 Newton MS, McLachlan HL, Willis KF, Forster DA. Comparing satisfaction and burnout between caseload and standard care midwives: findings from two cross-sectional surveys conducted in Victoria, Australia. BMC Pregnancy Childbirth 2014; 14: 426. doi: 10.1186/s12884-014-0426-7

7 Bloxsome D, Ireson D, Doleman G, Bayes S. Factors associated with midwives' job satisfaction and intention to stay in the profession: An integrative review. J Clin Nurs 2019; 28:386-399. doi: 10.1111/jocn.14651

8 Benjamin Y, Walsh D, Taub N. A comparison of partnership caseload midwifery care with conventional team midwifery care: labour and birth outcomes. Midwifery 2001 ; 17 : 234240. doi: $\underline{10.1054 / \mathrm{midw} .2001 .0257}$

9 Forster DA, McLachlan HL, Davey M-A, Biro MA, Farrell T, Gold L, Flood M, Shafiei T, Waldenström $U$. Continuity of care by a primary midwife (caseload midwifery) increases women's satisfaction with antenatal, intrapartum and postpartum care: results from the COSMOS randomised controlled trial. BMC Pregnancy Childbirth 2016; 16: 28. doi: 10.1186/s12884-016-0798-y

10 Ryan P, Revill P, Devane D, Normand C. An assessment of the cost-effectiveness of midwife-led care in the United Kingdom. Midwifery 2013; 29: 368-376. doi:

10.1016/j.midw.2012.02.005

11 Tracy SK, Hartz DL, Tracy MB, Allen J, Forti A, Hall B, White J, Lainchbury A, Stapleton H, Beckmann M, Bisits A, Homer C, Foureur M, Welsh A, Kildea S. Caseload midwifery care versus standard maternity care for women of any risk: M@NGO, a randomised controlled trial. Lancet 2013; 382: 1723-1732. doi: 10.1016/S0140-6736(13)61406-3

12 Eissler, A.B., Jerg-Bretzke, L. Der Arbeitsort beeinflusst die Belastungsfaktoren teilweise signifikant. Hebamme.ch 2015; Im Internet:

https://obstetrica.hebamme.ch/de/profiles/3f957b8ee011-obstetrica/editions/shv_fssf_fsl9-2015/pages; Stand: 21.08.2019 
13 Chien W-T, Yick S-Y. An Investigation of Nurses' Job Satisfaction in a Private Hospital and Its Correlates. Open Nurs J 2016; 10: 99-112. doi: 10.2174/1874434601610010099

14 SHV. Berufsdefinition der Hebamme. 2007; Im Internet: https://www.hebamme.ch/wpcontent/uploads/2018/06/01_Berufsdefinition-der-Hebamme-d.pdf; Stand: 20.02.2019

15 Merçay C, Burla L, Widmer M. Gesundheitspersonal in der Schweiz Bestandesaufnahme und Prognosen bis 2030. 2016; Im Internet:

https://www.obsan.admin.ch//sites/default/files/publications/2016/obsan_71_bericht.pdf; Stand: 20.02 .2019

16 Gerst T, Hibbeler B. Gesundheitsfachberufe: Auf dem Weg in die Akademisierung. Deutsches Ärzteblatt 2012; 109: 2458-2461; Im Internet:

https://www.aerzteblatt.de/archiv/133313/Gesundheitsfachberufe-Auf-dem-Weg-in-dieAkademisierung; Stand: 20.02.2019

17 Deutscher Wissenschaftsrat. Empfehlungen zu hochschulischen Qualifikationen für das Gesundheitswesen. 2012; Im Internet: https://www.wissenschaftsrat.de/download/archiv/2411-12.pdf; Stand: 20.02.2019

18 Maillefer F, de Labrusse C, Cardia-Vonèche L, Hohlfeld P, Stoll B. Women and healthcare providers' perceptions of a midwife-led unit in a Swiss university hospital: a qualitative study. BMC Pregnancy Childbirth 2015; 15: 56. doi: 10.1186/s12884-015-0477-4

19 Kantonsrat Zürich. Auszug aus dem Protokoll des Regierungsrates des Kantons Zürich Sitzung vom 20. Mai 2015, 551. Postulat (Stärkung der hebammengeleiteten Geburtshilfe im Kanton Zürich). 2015; Im Internet:

https://www.kantonsrat.zh.ch/Dokumente/Da2a819ac-0ffd-4ba3-b9c5-

3d9d0716e555/R15091.pdf; Stand: 20.02.2019

20 Deutsches Netzwerk für Qualitätsentwicklung in der Pflege (DNQP), Verbund

Hebammenforschung. Expertinnenstandard Förderung der physiologischen Geburt. 2014; Hochschule Osnabrück, Fakultät für Wirtschafts- und Sozialwissenschaften, OsnabrückInternet:

https://www.hebammenforschung.de/fileadmin/HSOS/Homepages/Hebammenforschung/ Geburt_Auszug.pdf; Stand: 20.02.2019

21 Bauer N, Sayn-Wittgenstein F zu, Verbund Hebammenforschung (Hrsg.). Handbuch Hebammenkreißsaal: von der Idee zur Umsetzung. Osnabrück: Verb. Hebammenforschung, 2007

22 van Teijlingen E, Hundley V. Pilot studies in family planning and reproductive health care. J Fam Plann Reprod Health Care 2005; 31: 219-221. doi: 10.1783/1471189054483735

23 Erdin, R, Grylka-Bäschlin, S, Schmid, M, Pehlke-Milde, J. Tätigkeitserfassung der frei praktizierenden Hebammen der Schweiz. 2017; Im Internet: https://digitalcollection.zhaw.ch/handle/11475/1577; Stand: 20.02.2019 


\section{Tabellenverzeichnis:}

Tabelle 1: $\quad$ Förderung der physiologischen Geburt und routinemässige Massnahmen in Einrichtungen mit und ohne hebammengeleiteten Geburten

Tabelle 2: Entscheidungsprozesse in Einrichtungen mit und ohne hebammengeleiteten Geburten

Tabelle 3: $\quad$ Selbständige und eigenverantwortliche Tätigkeiten der Hebammen in Einrichtungen mit und ohne hebammengeleiteten Geburten

Tabelle 4: Verantwortlichkeiten beim Austritt in Einrichtungen mit und ohne hebammengeleiteten Geburten 
Tabelle 1: Förderung der physiologischen Geburt und routinemässige Massnahmen in Einrichtungen mit und ohne hebammengeleiteten Geburten

\begin{tabular}{|c|c|c|c|c|}
\hline Statement & $\begin{array}{l}\text { Ganze Stich- } \\
\text { probe } \\
n=16\end{array}$ & $\begin{array}{l}\text { Mit hebam- } \\
\text { mengeleiteten } \\
\text { Geburten } \\
n=5\end{array}$ & $\begin{array}{l}\text { Ohne hebam- } \\
\text { mengeleitete } \\
\text { Geburten } \\
n=11\end{array}$ & p-Wert \\
\hline \multicolumn{5}{|c|}{ Förderung der physiologischen Geburt } \\
\hline $\begin{array}{l}\text { Übergaben zwischen den } \\
\text { Hebammen bei } \\
\text { Schichtwechsel finden } \\
\text { unter Einbeziehung der } \\
\text { Frau statt. }\end{array}$ & & & & 0.500 \\
\hline $\begin{array}{l}\text { Voll und ganz/ } \\
\text { grösstenteils, n (\%) }\end{array}$ & $8(50.0)$ & $3(60.0)$ & $5(45.5)$ & \\
\hline Nur zum Teil, nicht, n (\%) & $8(50.0)$ & $2(40.0)$ & $6(54.6)$ & \\
\hline \multicolumn{5}{|l|}{$\begin{array}{l}\text { Die Hebamme fördert die } \\
\text { Möglichkeit der Frau ihre } \\
\text { Geburtsposition frei zu } \\
\text { wählen, vorausgesetzt, } \\
\text { dass die medizinische } \\
\text { Situation dies erlaubt. }\end{array}$} \\
\hline $\begin{array}{l}\text { Voll und ganz/ } \\
\text { grösstenteils, n (\%) }\end{array}$ & $16(100)$ & $5(100)$ & $11(100)$ & \\
\hline Nur zum Teil, nicht, n (\%) & 0 & 0 & 0 & \\
\hline $\begin{array}{l}\text { Die Frau hat jederzeit die } \\
\text { Möglichkeit ihr Kind im } \\
\text { Wasser zu gebären, } \\
\text { vorausgesetzt, dass die } \\
\text { medizinische Situation } \\
\text { dies erlaubt. }\end{array}$ & & & & 0.687 \\
\hline $\begin{array}{l}\text { Voll und ganz/ } \\
\text { grösstenteils, n (\%) }\end{array}$ & $15(93.8)$ & $5(100.0)$ & $10(90.9)$ & \\
\hline Nur zum Teil, nicht, n (\%) & $1(6.25)$ & 0 & $1(9.1)$ & \\
\hline \multicolumn{5}{|l|}{$\begin{array}{l}\text { Alle Hebammen der } \\
\text { Institution gewährleisten } \\
\text { eine grösstmögliche } \\
\text { Mobilität der Frauen unter } \\
\text { der Geburt. }\end{array}$} \\
\hline $\begin{array}{l}\text { Voll und ganz/ } \\
\text { grösstenteils, n (\%) }\end{array}$ & $16(100)$ & $5(100)$ & $11(100)$ & \\
\hline Nur zum Teil, nicht, n (\%) & 0 & 0 & 0 & \\
\hline \multicolumn{5}{|c|}{ Routinemässige Massnahmen bei physiologischen Geburten } \\
\hline Venöser Zugang, n (\%) & $10(62.5)$ & $1(20.0)$ & $9(81.8)$ & 0.036 \\
\hline $\begin{array}{l}\text { Blutabnahme für Labor, } \\
\mathrm{n}(\%)\end{array}$ & $12(75.0)$ & $2(40.0)$ & $10(90.9)$ & 0.063 \\
\hline Aufnahme-CTG ${ }^{1}$, n (\%) & $14(87.5)$ & $3(60.0)$ & $11(100.0)$ & 0.083 \\
\hline
\end{tabular}




\begin{tabular}{|l|lll|l|}
$\begin{array}{l}\text { Kontinuierliches CTG } \\
\mathrm{n}(\%)\end{array}$ & $4(25.0)$ & $1(20.0)$ & $3(27.3)$ & 0.635 \\
$\begin{array}{l}\text { Ultraschall bei Eintritt, } \\
\mathrm{n}(\%)\end{array}$ & $8(50.0)$ & $1(20.0)$ & $7(63.6)$ & 0.141 \\
$\begin{array}{l}\text { Aufnahmeuntersuchung } \\
\text { Arzt/Ärztin, n (\%) } \\
\begin{array}{l}\text { Oxytocin in Plazentar- } \\
\text { periode, } \mathrm{n}(\%)\end{array}\end{array}$ & $6(37.5)$ & 0 & $6(54.6)$ & 0.058 \\
\hline
\end{tabular}

${ }^{1} \mathrm{CTG}=$ Kardiotokographie

${ }^{2}$ während Eröffnungs- oder Austreibungsphase 
Tabelle 2: Entscheidungsprozesse in Einrichtungen mit und ohne hebammengeleiteten Geburten

\begin{tabular}{|c|c|c|c|}
\hline $\begin{array}{l}\text { Massnahme/ } \\
\text { Entscheidungsprozess }\end{array}$ & $\begin{array}{l}\text { Mit hebammen- } \\
\text { geleiteten } \\
\text { Geburten, n=5 }\end{array}$ & $\begin{array}{l}\text { Ohne hebam- } \\
\text { mengeleitete } \\
\text { Geburten, } n=11\end{array}$ & p-Wert \\
\hline $\begin{array}{l}\text { Die Gebärende in der Latenzphase } \\
\text { kehrt nach Hause zurück } \\
\text { Gebärende, n (\%) } \\
\text { Hebamme, n (\%) } \\
\text { Verordnung Arzt/Ärztin, n (\%) } \\
\text { Gebärende \& Hebamme, n (\%) } \\
\text { Gebärende \& Arzt/Ärztin, n (\%) } \\
\text { Hebamme \& Arzt/Ärztin, n (\%) } \\
\text { Gebärende, Hebamme \& } \\
\text { Arzt/Ärztin, n (\%) } \\
\text { Situationsabhängig, n (\%) } \\
\text { Es gibt keine klare Regelung, n (\%) } \\
\text { Nicht anwendbar, n (\%) }\end{array}$ & $\begin{array}{l}1(20.0) \\
2(40.0) \\
1(20.0) \\
1(20.0)\end{array}$ & $\begin{array}{l}1(9.1) \\
1(9.1) \\
1(9.1) \\
8(72.7)\end{array}$ & 0.013 \\
\hline $\begin{array}{l}\text { Die Gebärende mit vorzeitigem } \\
\text { Blasensprung kehrt nach Hause } \\
\text { zurück }^{1} \\
\text { Gebärende, n (\%) } \\
\text { Hebamme, n (\%) } \\
\text { Verordnung Arzt/Ärztin, n (\%) } \\
\text { Gebärende \& Hebamme, n (\%) } \\
\text { Gebärende \& Arzt/Ärztin, n (\%) } \\
\text { Hebamme \& Arzt/Ärztin, n (\%) } \\
\text { Gebärende, Hebamme \& } \\
\text { Arzt/Ärztin, n (\%) } \\
\text { Situationsabhängig, n (\%) } \\
\text { Es gibt keine klare Regelung, n (\%) } \\
\text { Nicht anwendbar, n (\%) }\end{array}$ & $\begin{array}{l}1(25.0) \\
1(25.0) \\
1(25.0) \\
1(25.0)\end{array}$ & $6(9.1)$ & 0.004 \\
\hline $\begin{array}{l}\text { Durchführung einer } \\
\text { Ultraschalluntersuchung } \\
\text { Gebärende, n (\%) } \\
\text { Hebamme, n (\%) } \\
\text { Verordnung Arzt/Ärztin, n (\%) } \\
\text { Gebärende \& Hebamme, n (\%) } \\
\text { Gebärende \& Arzt/Ärztin, n (\%) } \\
\text { Hebamme \& Arzt/Ärztin, n (\%) } \\
\text { Gebärende, Hebamme \& } \\
\text { Arzt/Ärztin, n (\%) } \\
\text { Situationsabhängig, n (\%) } \\
\text { Es gibt keine klare Regelung, n (\%) } \\
\text { Nicht anwendbar, n (\%) }\end{array}$ & $\begin{array}{l}2(40.0) \\
1(20.0)\end{array}$ & $\begin{array}{l}6(54.6) \\
2(18.2)\end{array}$ & 0.072 \\
\hline
\end{tabular}




\begin{tabular}{|c|c|c|c|}
\hline $\begin{array}{l}\text { Mobilität unter der Geburt } \\
\text { Gebärende, n (\%) } \\
\text { Hebamme, n (\%) } \\
\text { Verordnung Arzt/Ärztin, n (\%) } \\
\text { Gebärende \& Hebamme, n (\%) } \\
\text { Gebärende \& Arzt/Ärztin, n (\%) } \\
\text { Hebamme \& Arzt/Ärztin, n (\%) } \\
\text { Gebärende, Hebamme \& } \\
\text { Arzt/Ärztin, n (\%) } \\
\text { Situationsabhängig, n (\%) } \\
\text { Es gibt keine klare Regelung, n (\%) } \\
\text { Nicht anwendbar, n (\%) }\end{array}$ & $\begin{array}{l}4(80.0) \\
1(20.0)\end{array}$ & $\begin{array}{l}1(9.1) \\
2(18.2) \\
8(72.7)\end{array}$ & 0.093 \\
\hline $\begin{array}{l}\text { Entspannungsbad } \\
\text { Gebärende, n (\%) } \\
\text { Hebamme, n (\%) } \\
\text { Verordnung Arzt/Ärztin, n (\%) } \\
\text { Gebärende \& Hebamme, n (\%) } \\
\text { Gebärende \& Arzt/Ärztin, n (\%) } \\
\text { Hebamme \& Arzt/Ärztin, n (\%) } \\
\text { Gebärende, Hebamme \& } \\
\text { Arzt/Ärztin, n (\%) } \\
\text { Situationsabhängig, n (\%) } \\
\text { Es gibt keine klare Regelung, n (\%) } \\
\text { Nicht anwendbar, n (\%) }\end{array}$ & $\begin{array}{l}1(20.0) \\
2(40.0) \\
2(40.0)\end{array}$ & $\begin{array}{l}1(9.1) \\
10(90.9)\end{array}$ & 0.063 \\
\hline $\begin{array}{l}\text { Beginn Wehenstimulation mit } \\
\text { Oxytocin } \\
\text { Gebärende, n (\%) } \\
\text { Hebamme, n (\%) } \\
\text { Verordnung Arzt/Ärztin, n (\%) } \\
\text { Gebärende \& Hebamme, n (\%) } \\
\text { Gebärende \& Arzt/Ärztin, n (\%) } \\
\text { Hebamme \& Arzt/Ärztin, n (\%) } \\
\text { Gebärende, Hebamme \& } \\
\text { Arzt/Ärztin, n (\%) } \\
\text { Situationsabhängig, n (\%) } \\
\text { Es gibt keine klare Regelung, n (\%) } \\
\text { Nicht anwendbar, n (\%) }\end{array}$ & $\begin{array}{l}2(40.0) \\
1(20.0)\end{array}$ & $\begin{array}{l}1(9.1) \\
1(9.1) \\
6(54.6) \\
2(18.2) \\
1(9.1)\end{array}$ & 0.098 \\
\hline
\end{tabular}

${ }^{1} \mathrm{n}=15$ 
Tabelle 3: Selbständige und eigenverantwortliche Tätigkeiten der Hebammen in Einrichtungen mit und ohne hebammengeleiteten Geburten

\begin{tabular}{|c|c|c|c|c|}
\hline $\begin{array}{l}\text { Selbständige und } \\
\text { selbstverantwortliche } \\
\text { Tätigkeit }\end{array}$ & $\begin{array}{l}\text { Ganze } \\
\text { Stichprobe, } \\
n=16\end{array}$ & $\begin{array}{l}\text { Einrichtungen } \\
\text { mit hebammen- } \\
\text { geleiteten } \\
\text { Geburten, } n=5\end{array}$ & $\begin{array}{l}\text { Einrichtungen } \\
\text { ohne hebam- } \\
\text { mengeleiteten } \\
\text { Geburten, } n=11\end{array}$ & p-Wert \\
\hline $\begin{array}{l}\text { Anamnese der Frau } \\
\text { Immer/meistens, n (\%) } \\
\text { Selten/nie, n (\%) }\end{array}$ & $\begin{array}{l}13(81.3) \\
3(18.8)\end{array}$ & $\begin{array}{l}5(100.0) \\
0\end{array}$ & $\begin{array}{l}8(72.7) \\
3(27.3)\end{array}$ & 0.295 \\
\hline $\begin{array}{l}\text { Klinisches Assessment } \\
\text { für die Aufnahme zur } \\
\text { Geburt } \\
\text { Immer/meistens, n (\%) } \\
\text { Selten/nie, n (\%) }\end{array}$ & $\begin{array}{l}13(81.3) \\
3(18.8)\end{array}$ & $\begin{array}{l}5(100.0) \\
0\end{array}$ & $\begin{array}{l}8(72.7) \\
3(27.3)\end{array}$ & 0.295 \\
\hline $\begin{array}{l}\text { Alternative Mass- } \\
\text { nahmen } \\
\text { Immer/meistens, } \mathrm{n}(\%) \\
\text { Selten/nie, } \mathrm{n}(\%)\end{array}$ & $\begin{array}{l}16(100.0) \\
0\end{array}$ & $\begin{array}{l}5(100.0) \\
0\end{array}$ & $\begin{array}{l}11(100.0) \\
0\end{array}$ & \\
\hline $\begin{array}{l}\text { Amniotomie } \\
\text { Immer/meistens, n (\%) } \\
\text { Selten/nie, n (\%) }\end{array}$ & $\begin{array}{l}14(87.5) \\
2(12.5)\end{array}$ & $\begin{array}{l}5(100.0) \\
0\end{array}$ & $\begin{array}{l}9(81.2) \\
2(18.2)\end{array}$ & 0.458 \\
\hline $\begin{array}{l}\text { Dammschutz } \\
\text { Immer/meistens, n (\%) } \\
\text { Selten/nie, n (\%) }\end{array}$ & $\begin{array}{l}15(93.8) \\
1(6.3)\end{array}$ & $\begin{array}{l}5(100.0) \\
0\end{array}$ & $\begin{array}{l}10(90.9) \\
1(9.1)\end{array}$ & 0.687 \\
\hline $\begin{array}{l}\text { Episiotomie schneiden } \\
\text { Immer/meistens, n (\%) } \\
\text { Selten/nie, n (\%) }\end{array}$ & $\begin{array}{l}7(43.8) \\
9(56.3)\end{array}$ & $\begin{array}{l}3(60.0) \\
2(40.0)\end{array}$ & $\begin{array}{l}4(36.4) \\
7(63.6)\end{array}$ & 0.365 \\
\hline $\begin{array}{l}\text { Dammverletzung, } \\
\text { Episiotomie nähen } \\
\text { Immer/meistens, n (\%) } \\
\text { Selten/nie, n (\%) }\end{array}$ & $\begin{array}{l}1(6.3) \\
15(93.8)\end{array}$ & $\begin{array}{l}1(20.0) \\
4(80.0)\end{array}$ & $\begin{array}{l}0 \\
11(100.0)\end{array}$ & 0.312 \\
\hline $\begin{array}{l}\text { Apgar-Score } \\
\text { Immer/meistens, n (\%) } \\
\text { Selten/nie, n (\%) }\end{array}$ & $\begin{array}{l}16(100.0) \\
0\end{array}$ & $\begin{array}{l}5(100.0) \\
0\end{array}$ & $\begin{array}{l}11(100.0) \\
0\end{array}$ & \\
\hline $\begin{array}{l}\text { Status Neugeborenes } \\
\text { Immer/meistens, n (\%) } \\
\text { Selten/nie, n (\%) }\end{array}$ & $\begin{array}{l}9(56.3) \\
7(43.8)\end{array}$ & $\begin{array}{l}3(60.0) \\
2(40.0)\end{array}$ & $\begin{array}{l}6(54.6) \\
5(45.5)\end{array}$ & 0.635 \\
\hline
\end{tabular}


Tabelle 4: Verantwortlichkeiten beim Austritt in Einrichtungen mit und ohne hebammengeleiteten Geburten

\begin{tabular}{|c|c|c|c|c|}
\hline $\begin{array}{l}\text { Verantwortlichkeiten } \\
\text { beim Austritt } \\
\text { Mehrfachnennungen } \\
\text { möglich }\end{array}$ & $\begin{array}{l}\text { Ganze } \\
\text { Stichprobe, } \\
n=16\end{array}$ & $\begin{array}{l}\text { Einrichtungen } \\
\text { mit hebammen- } \\
\text { geleiteten } \\
\text { Geburten, } n=5\end{array}$ & $\begin{array}{l}\text { Einrichtungen } \\
\text { ohne hebam- } \\
\text { mengeleiteten } \\
\text { Geburten, } n=11\end{array}$ & p-Wert \\
\hline $\begin{array}{l}\text { Austrittgespräch } \\
\text { Hebamme/Pflegefach- } \\
\text { person, n (\%) } \\
\text { Arzt/Ärztin, n (\%) }\end{array}$ & $\begin{array}{l}16(100) \\
8(50.0)\end{array}$ & $\begin{array}{l}5(100.0) \\
2(40.0)\end{array}$ & $\begin{array}{l}11(100.0) \\
6(54.6)\end{array}$ & 0.500 \\
\hline $\begin{array}{l}\text { Austrittsuntersuchung } \\
\text { Hebamme/Pflegefach- } \\
\text { person, n (\%) } \\
\text { Arzt/Ärztin, n (\%) }\end{array}$ & $\begin{array}{l}4(25.0) \\
12(75.0)\end{array}$ & $\begin{array}{l}3(60.0) \\
2(40.0)\end{array}$ & $\begin{array}{l}1(9.1) \\
10(90.9)\end{array}$ & $\begin{array}{l}0.063 \\
0.063\end{array}$ \\
\hline $\begin{array}{l}\text { Vermittlung ambulante } \\
\text { Wochenbettbetreuung } \\
\text { Hebamme/Pflegefach- } \\
\text { person, n (\%) } \\
\text { Arzt/Ärztin, n (\%) }\end{array}$ & $\begin{array}{l}16(100.0) \\
0\end{array}$ & $\begin{array}{l}5(100.0) \\
0\end{array}$ & $\begin{array}{l}11(100.0) \\
0\end{array}$ & \\
\hline $\begin{array}{l}\text { Entlassungsbrief nach- } \\
\text { betreuende Hebamme } \\
\text { Hebamme/Pflegefach- } \\
\text { person, n (\%) } \\
\text { Arzt/Ärztin, n (\%) }\end{array}$ & $\begin{array}{l}15(93.8) \\
1(6.3)\end{array}$ & $\begin{array}{l}5(100.0) \\
0\end{array}$ & $\begin{array}{l}10(90.9) \\
1(9.1)\end{array}$ & $\begin{array}{l}0.687 \\
0.687\end{array}$ \\
\hline $\begin{array}{l}\text { Organisation Unter- } \\
\text { stützung psycho- } \\
\text { soziale Risikofaktoren } \\
\text { Hebamme/Pflegefach- } \\
\text { person, n (\%) } \\
\text { Arzt/Ärztin, n (\%) }\end{array}$ & $\begin{array}{l}11(68.8) \\
13(81.3)\end{array}$ & $\begin{array}{l}4(80.0) \\
3(60.0)\end{array}$ & $\begin{array}{l}7(63.6) \\
10(90.9)\end{array}$ & $\begin{array}{l}0.484 \\
0.214\end{array}$ \\
\hline
\end{tabular}

